\title{
Sepsis-related Mortality Among Adults Aged 65 and Over: United States, 2019
}

\author{
Ellen A. Kramarow, Ph.D.
}

\section{Key findings}

\section{Data from the National Vital Statistics System}

- Sepsis-related death rates for adults aged 65 and over varied from 2000 through 2019 but generally declined over this period.

- Among adults aged 65 and over, sepsis-related death rates in 2019 increased with age; rates were about five times higher among adults aged 85 and over $(750.0$ per 100,000$)$ compared with adults aged 65-74 (150.7).

- In 2019, sepsis-related death rates for adults aged 65 and over were highest among non-Hispanic black adults (377.4 per 100,000) compared with non-Hispanic white (275.7), non-Hispanic Asian (180.0), and Hispanic (246.4) adults.

- Among adults aged 65 and over, sepsis-related death rates in 2019 were higher in rural areas compared with urban areas.
Sepsis is the body's extreme response to an infection and, if not identified and treated quickly, may lead to serious medical consequences and death (1). Sepsis can occur at any age, but infants, people with chronic conditions, people with weakened immune systems, and older adults are at high risk (1). In 2019, there were 201,092 deaths in the United States involving sepsis, with three-fourths of those deaths occurring among persons aged 65 and over (2). This report describes sepsis-related mortality among adults aged 65 and over by age, sex, race and Hispanic origin, and urbanicity.

\section{From 2000-2019, changes in sepsis-related death rates among adults aged 65 and over varied by age group.}

- Sepsis-related death rates for adults aged 65 and over declined between 2000 (298.8 per 100,000) and 2019 (277.4), with periods of both decline and stability during this time (Figure 1).

Figure 1. Sepsis-related death rates for adults aged 65 and over, by age group: United States, 2000-2019

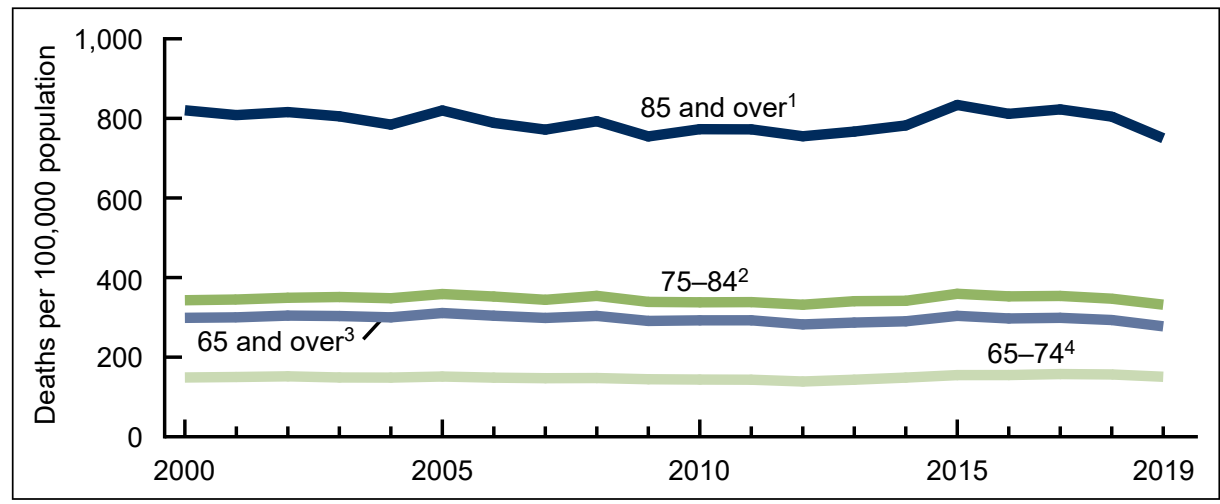

${ }^{1}$ Significant decreasing trend from 2000-2012; significant increasing trend from 2012-2017; and stable trend from 2017-2019 $(p<0.05)$.

2Stable trend from 2000-2005; significant decreasing trend from 2005-2012; stable trend from 2012-2016; and significant decreasing trend from 2016-2019 $(p<0.05)$.

${ }^{3}$ Stable trend from 2000-2005; significant decreasing trend from 2005-2012; stable trend from 2012-2017; and significant decreasing trend from $2017-2019(p<0.05)$.

${ }^{4}$ Stable trend from 2000-2005; significant decreasing trend from 2005-2012; significant increasing trend from 2012-2017; and stable trend from 2017-2019 $(p<0.05)$

NOTES: Sepsis-related deaths are those with sepsis or septicemia, International Classification of Diseases codes A40-A41, reported anywhere on the death certificate. For mortality statistics, sepsis and septicemia are synonymous and used interchangeably for classification purposes. Access data table for Figure 1 at: https://www.cdc.gov/nchs/data/databriefs/ db422-tables.pdf\#1.

SOURCE: National Center for Health Statistics, National Vital Statistics System, Mortality.

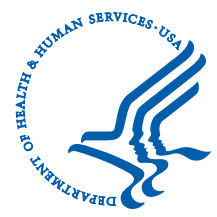




\section{NCHS Data Brief No. 422 November 2021}

- Sepsis-related death rates for adults aged 65-74 remained relatively stable between 2000 and 2019, with some offsetting decline and increase during the period. The rate for adults aged 65-74 was 150.7 in 2019.

- Sepsis-related death rates for adults aged 75-84 followed the same pattern as all adults aged 65 and over and declined from 343.0 in 2000 to 331.8 in 2019.

- While variable over time, sepsis-related death rates for adults aged 85 and over generally declined between 2000 (820.3) and 2019 (750.0).

- From 2000 through 2019, sepsis-related death rates for adults aged 85 and over were at least five times higher than rates for adults aged 65-74 and two times higher than rates for adults aged $75-84$.

\section{Sepsis-related death rates increased with age among adults aged 65 and over.}

- In 2019 , sepsis-related deaths increased from 150.7 per 100,000 among those aged 65-74 to 331.8 among those aged $75-84$ to 750.0 among those aged 85 and over (Figure 2).

- Among adults aged 65 and over, males had higher sepsis-related death rates than females overall (306.0 compared with 254.5).

Figure 2. Sepsis-related death rates for adults aged 65 and over, by age group and sex: United States, 2019

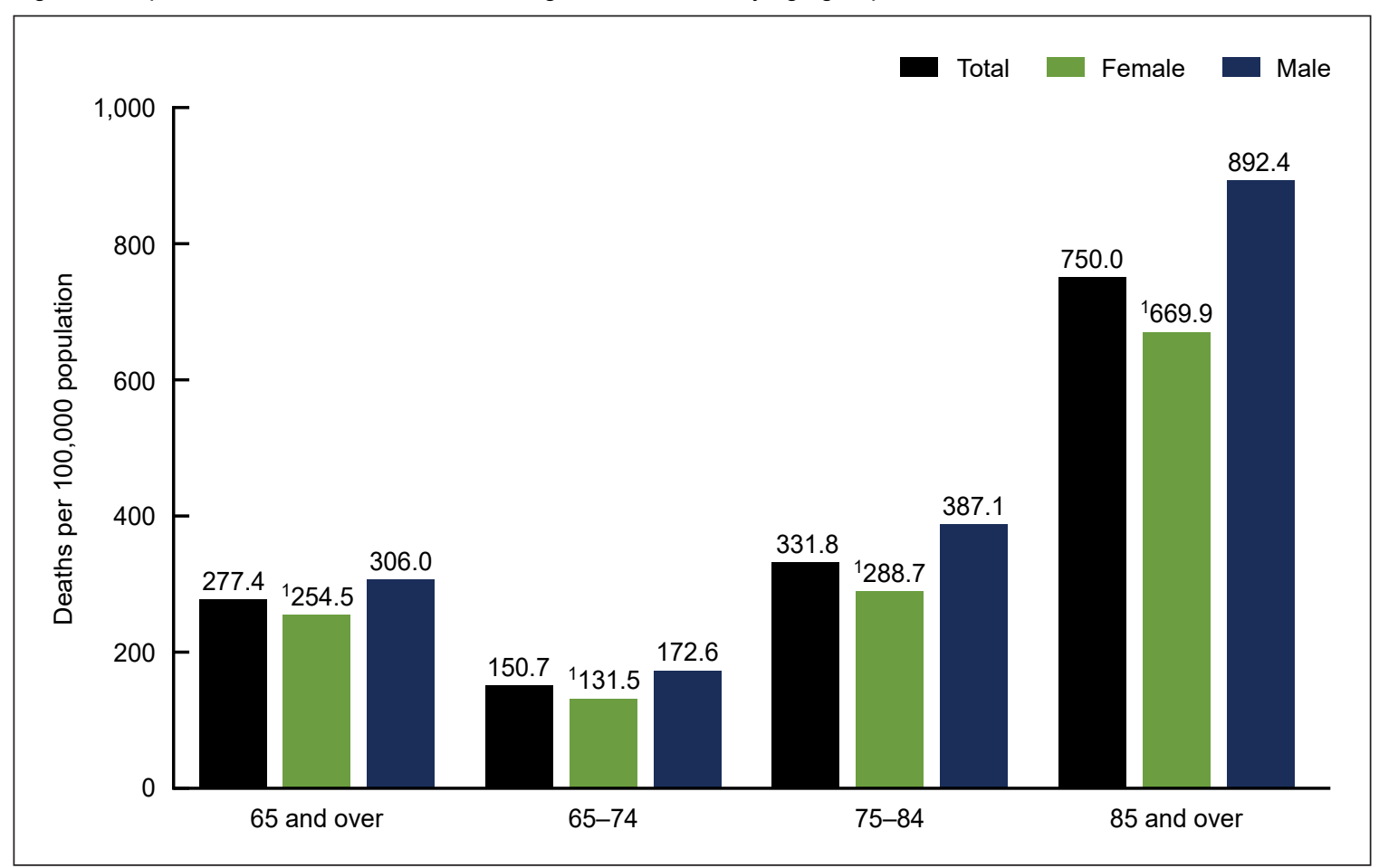

${ }^{1}$ Significantly lower than rates for males $(p<0.05)$

NOTES: Significant linear trend by age group for total, females and males $(p<0.05)$. Sepsis-related deaths are those with sepsis or septicemia, International Classification of Diseases codes A40-A41, reported anywhere on the death certificate. For mortality statistics, sepsis and septicemia are synonymous and used interchangeably for classification purposes. Access data table for Figure 2 at: https://www.cdc.gov/nchs/data/databriefs/db422-tables.pdf\#2.

SOURCE: National Center for Health Statistics, National Vital Statistics System, Mortality. 


\section{NCHS Data Brief a No. 422 a November 2021}

- Sepsis-related death rates for males were higher than rates for females among adults aged 65-74 (172.6 compared with 131.5), adults aged 75-84 (387.1 compared with 288.7), and adults aged 85 and over (892.4 compared with 669.9).

\section{Sepsis-related death rates among adults aged 65 and over were higher among non-Hispanic black adults than among other race and Hispanic- origin groups.}

- Among adults aged 65 and over, sepsis-related death rates were highest among non-Hispanic black adults (377.4 per 100,000), followed by non-Hispanic white (275.7), Hispanic (246.4), and non-Hispanic Asian (180.0) adults (Figure 3).

- Sepsis-related death rates were also highest among non-Hispanic black adults compared with other race and Hispanic-origin groups for those aged 65-74 (245.2), 75-84 (473.7), and 85 and over (921.3).

- Sepsis-related death rates increased with age among all race and Hispanic-origin groups.

Figure 3. Sepsis-related death rates for adults aged 65 and over, by age group and race and Hispanic origin: United States, 2019

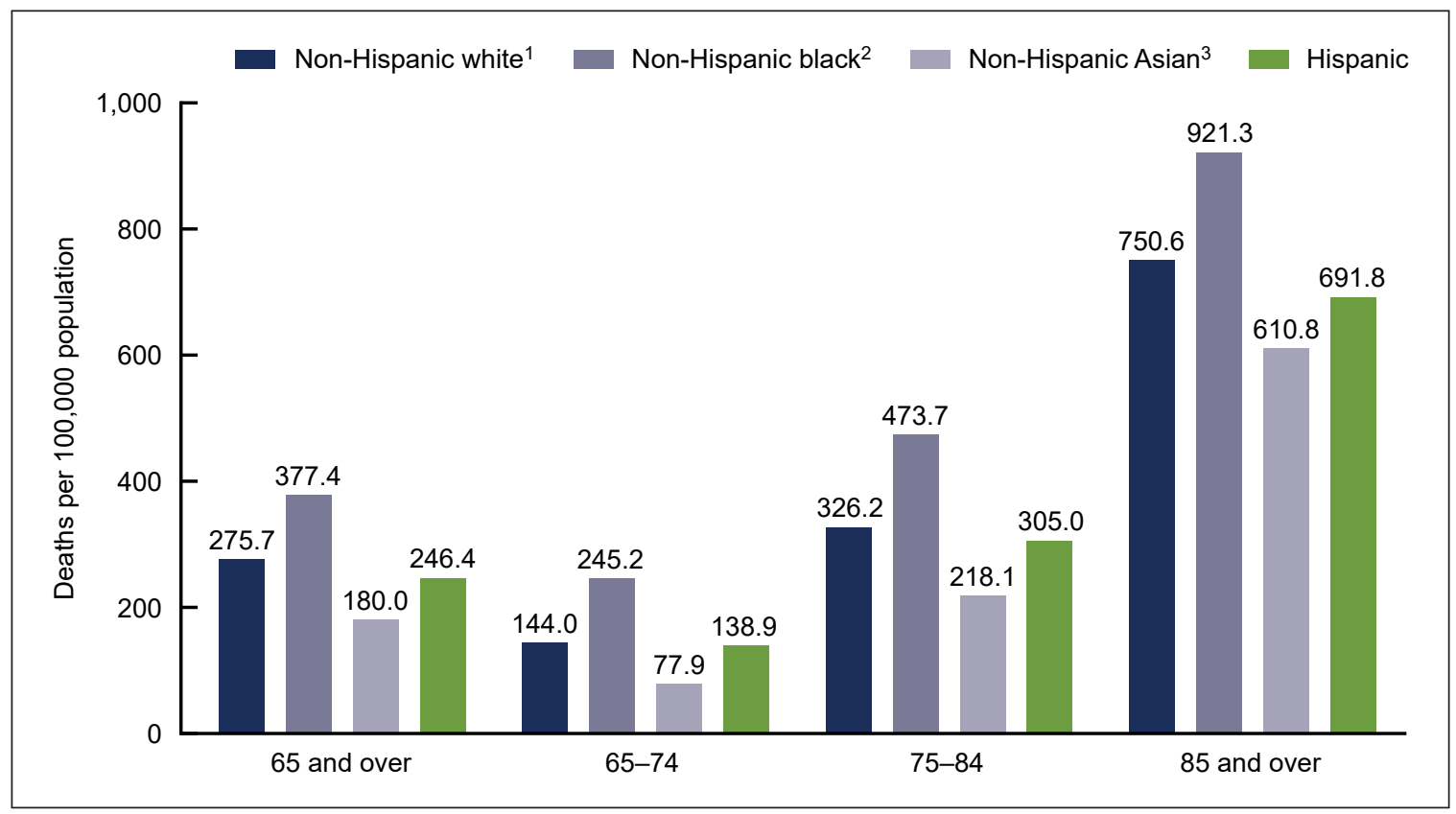

${ }^{1}$ Significantly lower than non-Hispanic black adults and significantly higher than non-Hispanic Asian and Hispanic adults $(p<0.05)$ ${ }^{2}$ Significantly higher than non-Hispanic Asian and Hispanic adults $(p<0.05)$

${ }^{3}$ Significantly lower than Hispanic adults $(p<0.05)$.

NOTES: Significant linear trend by age group for all race and Hispanic-origin groups $(p<0.05)$. Estimates are based on single-race data. Sepsis-related deaths are those with sepsis or septicemia, International Classification of Diseases codes A40-A41, reported anywhere on the death certificate. For mortality statistics, sepsis and septicemia are synonymous and used interchangeably for classification purposes. Access data table for Figure 3 at: https://www.cdc.gov/nchs/data/ databriefs/db422-tables.pdf\#3.

SOURCE: National Center for Health Statistics, National Vital Statistics System, Mortality. 


\section{Sepsis-related death rates among adults aged 65 and over were higher in rural areas compared with urban areas.}

- Among adults aged 65 and over, sepsis-related death rates were higher in rural areas (307.3 per 100,000) compared with urban areas (271.3) (Figure 4).

- Death rates were higher in rural areas than urban areas for age groups 65-74 (175.6 compared with 145.7), 75-84 (365.6 compared with 324.7), and 85 and over (801.2 compared with 739.9).

- Sepsis-related death rates increased with age in both urban and rural areas.

Figure 4. Sepsis-related death rates for adults aged 65 and over, by age group and urbanicity: United States, 2019

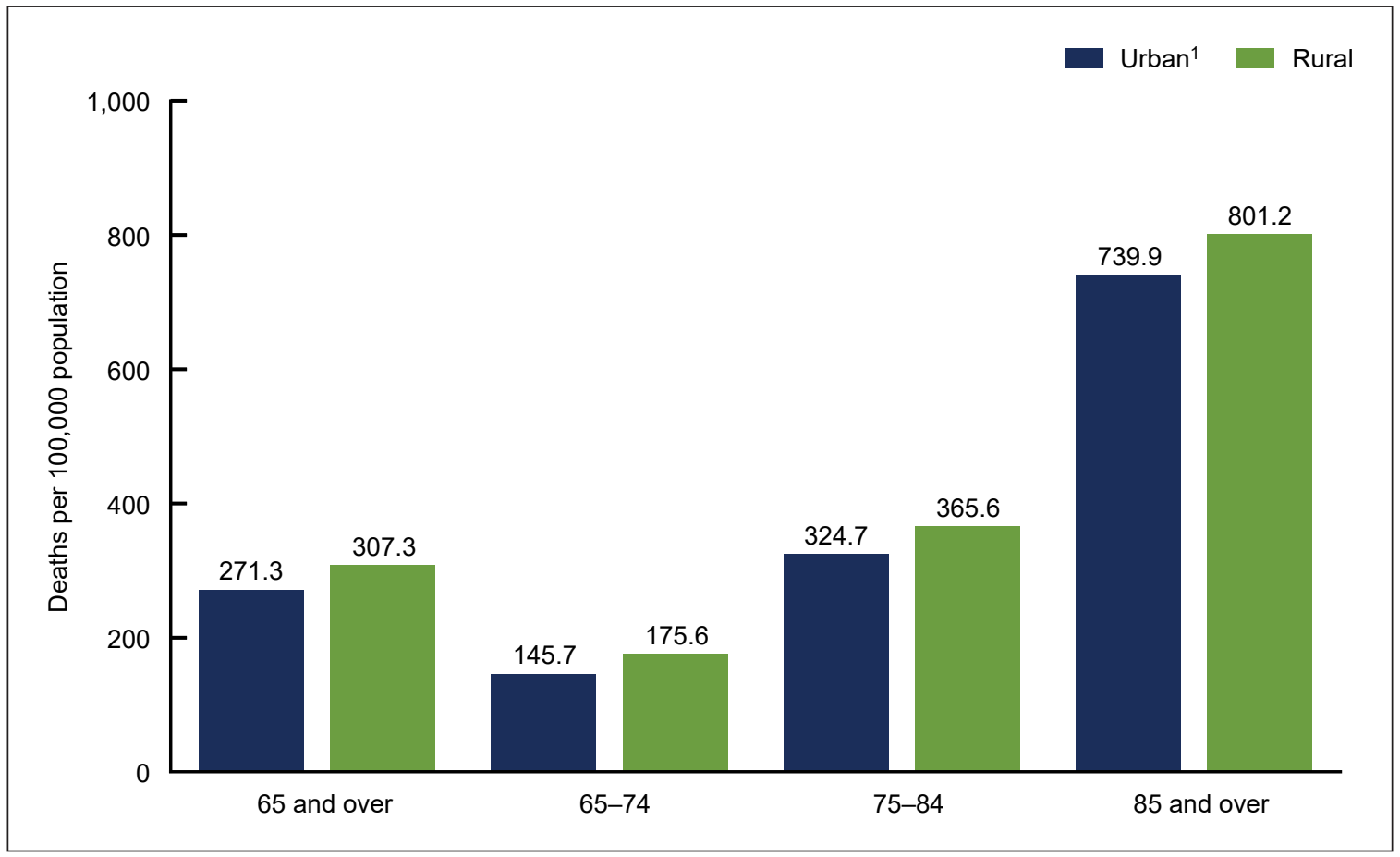

${ }^{1}$ Significantly lower than rates for rural populations for all age groups $(p<0.05)$

NOTES: Significant linear trend by age group for urban and rural areas $(p<0.05)$. Sepsis-related deaths are those with sepsis or septicemia, International

Classification of Diseases codes A40-A41, reported anywhere on the death certificate. For mortality statistics, sepsis and septicemia are synonymous and used interchangeably for classification purposes. Access data table for Figure 4 at: https://www.cdc.gov/nchs/data/databriefs/db422-tables.pdf\#4.

SOURCE: National Center for Health Statistics, National Vital Statistics System, Mortality. 


\section{NCHS Data Brief " No. 422 - November 2021}

\section{Summary}

In 2019, the sepsis-related death rate among adults aged 65 and over was 277.4 per 100,000. Death rates increased with age and were about five times higher among adults aged 85 and over (750.0) compared with adults aged 65-74 (150.7). Trends in sepsis-related death rates among adults aged 65 and over varied from 2000 through 2019, overall and by age group. Death rates were higher for males than females and for adults in rural compared with urban areas. Sepsisrelated death rates for non-Hispanic black adults were higher than those for other race and Hispanic-origin groups. Deaths among adults aged 65 and over accounted for approximately $75 \%$ of all sepsis-related deaths in the United States in 2019 (2).

Sepsis is typically a complication of another condition and is usually correctly reported as the immediate or as an intermediate cause of death on the death certificate (3). However, in 2019, $19.4 \%$ of all sepsis-related deaths among adults aged 65 and over were attributed to sepsis as the underlying cause of death (2). Death rates for sepsis may be affected by changes in diagnosis, treatment, and administrative coding, in addition to changes in underlying rates of disease and infection (4). Efforts to improve diagnosis of sepsis in a variety of healthcare settings to enhance care and prevent death are ongoing (5). 


\section{Data source and methods}

Estimates in this report are based on the National Vital Statistics System multiple cause of death files, accessed via CDC WONDER (2). Sepsis-related deaths were defined as having International Classification of Diseases, 10th Revision (ICD-10) (6) codes A40-A41 anywhere on the death certificate, either as an immediate, intermediate, underlying, or contributing cause of death. Some commonly reported underlying causes for sepsis-related deaths among adults aged 65 and over in 2019 were cancer, influenza and pneumonia, and heart disease (2). Sepsis will be selected as the underlying cause in cases where the actual underlying cause is not reported. Examining death rates calculated from deaths where sepsis is recorded anywhere on the death certificate better captures the burden of sepsis mortality than relying on underlying cause-of-death statistics alone.

Urbanicity was categorized using the 2013 NCHS Urban-Rural Classification Scheme for Counties (7). Counties were classified into six urbanization levels based on metropolitannonmetropolitan status, population distribution, and other factors. The four metropolitan categories (large central metro, large fringe metro, medium metro, and small metro) were grouped as urban counties. The two nonmetropolitan categories (micropolitan and noncore) were grouped as rural counties.

Trends in death rates were evaluated using the Joinpoint Regression Program (Version 4.8.1.0) (8). Joinpoint software was used to fit weighted least-squares regression models to the rates on the log transform scale. Analyses were set to allow a maximum of three joinpoints across the period, a minimum of three observed time points from any given joinpoint to either end of the data, and a minimum of four observed time points between any two joinpoints. The permutation tests for model (number of joinpoints) significance were set at an overall alpha level of 0.05 (9). Pairwise comparisons of rates were conducted using a two-sided $z$ test with an alpha level of $0.05(10)$.

Estimates for 2019 by race and Hispanic origin are based on the 1997 Office of Management and Budget standards for collection of race and Hispanic ethnicity data. Estimates for non-Hispanic white adults, non-Hispanic black adults, and non-Hispanic Asian adults are for those deaths where only one race was identified on the death certificate. Additional details on race and Hispanicorigin categories are available elsewhere (10).

\section{About the author}

Ellen A. Kramarow is with the National Center for Health Statistics, Division of Analysis and Epidemiology. 


\section{NCHS Data Brief " No. 422 - November 2021}

\section{References}

1. Centers for Disease Control and Prevention. What is sepsis? 2021. Available from: https://www.cdc.gov/sepsis/what-is-sepsis.html.

2. National Center for Health Statistics. About multiple cause of death, 1999-2019. CDC WONDER online database. 2020. Available from: https://wonder.cdc.gov/mcd-icd10.html.

3. Flagg LA, Anderson RN. Unsuitable underlying causes of death for assessing the quality of cause-of-death reporting. National Vital Statistics Reports; vol 69 no 14. Hyattsville, MD: National Center for Health Statistics. 2021.

4. Epstein L, Dantes R, Magill S, Fiore A. Varying estimates of sepsis mortality using death certificates and administrative codes-United States, 1999-2014. MMWR Morb Mortal Wkly Rep 65(13):342-5. 2016. DOI: 10.15585/mmwr.mm6513a2.

5. National Academies of Sciences, Engineering, and Medicine. Achieving excellence in sepsis diagnosis: Proceedings of a workshop in brief. Washington, DC: The National Academies Press. 2020. Available from: https://doi.org/10.17226/26034.

6. World Health Organization. International statistical classification of diseases and related health problems, 10th revision (ICD-10). 2008 ed. Geneva, Switzerland. 2009.

7. Ingram DD, Franco SJ. 2013 NCHS urban-rural classification scheme for counties. National Center for Health Statistics. Vital Health Stat 2(166). 2014. Available from: https://www.cdc.gov/ nchs/data/series/sr_02/sr02_166.pdf.

8. National Cancer Institute. Joinpoint regression program (Version 4.8.0.1) [computer software]. 2021.

9. Ingram DD, Malec DJ, Makuc DM, Kruszon-Moran D, Gindi RM, Albert M, et al. National Center for Health Statistics guidelines for analysis of trends. National Center for Health Statistics. Vital Health Stat 2(179). 2018.

10. Xu JQ, Murphy SL, Kochanek KD, Arias E. Deaths: Final data for 2019. National Vital Statistics Reports; vol 70 no 08. Hyattsville, MD: National Center for Health Statistics. 2021. DOI: https://dx.doi.org/10.15620/cdc:106058. 
Centers for Disease Control and Prevention

\section{NCHS Data Brief a No. 422 - November 2021}

Keywords: septicemia $\bullet$ death rates $\bullet$ urban or rural $\bullet$ race and ethnicity $\bullet$ National Vital Statistics System

\section{Suggested citation}

Kramarow EA. Sepsis-related mortality among adults aged 65 and over:

United States, 2019. NCHS Data Brief, no 422. Hyattsville, MD: National Center for Health Statistics. 2021. DOI: https://dx.doi.org/10.15620/cdc:110542.

\section{Copyright information}

All material appearing in this report is in the public domain and may be reproduced or copied without permission; citation as to source, however, is appreciated.

\section{National Center for Health Statistics}

Brian C. Moyer, Ph.D., Director Amy M. Branum, Ph.D., Associate Director for Science

Division of Analysis and Epidemiology Irma E. Arispe, Ph.D., Director Kevin C. Heslin, Ph.D., Associate Director for Science

For e-mail updates on NCHS publication releases, subscribe online at: https://www.cdc.gov/nchs/email-updates.htm.

For questions or general information about NCHS:

Tel: 1-800-CDC-INFO (1-800-232-4636)

TTY: $1-888-232-6348$

Internet: https://www.cdc.gov/nchs

Online request form: https://www.cdc.gov/info

ISSN 1941-4927 Print ed.

ISSN 1941-4935 Online ed. 